\title{
Voice Disorders after Total Thyroidectomy: Prospective Evaluation by Patient Self-Assessment, Indirect Laryngoscopy and Ultrasonography
}

\author{
Alexander M. Shulutko ${ }^{1}$. Vasiliy I. Semikov ${ }^{1}$. Andrey Y. Moiseev ${ }^{1}$. Elkhan G. Osmanov ${ }^{1}$. Julia A. Boblak ${ }^{1}$. \\ Alla R. Patalova ${ }^{1} \cdot$ Gaukhar T. Mansurova $^{1,2}$ - Airazat M. Kazaryan ${ }^{1,3,4,5,6}$
}

Received: 4 October 2021 / Accepted: 19 February 2022

(c) The Author(s) 2022

\begin{abstract}
Voice alterations after thyroidectomy with mobile vocal folds are common. Ultrasonography has been used to assess the mobility of the vocal folds after thyroidectomy. Fifty-four patients underwent thyroidectomy. Indirect laryngoscopy, ultrasonography, and GRBAS (grade, roughness, breathiness, asthenia, strain) scoring were performed preoperatively, 3 days, 2 and 6 months, postoperatively. On the third postoperative day, the mobility of the vocal folds was preserved in 52 patients and pareses were recorded in 2 patients. All patients after thyroidectomy noted the presence of voice alteration in the absence of the postoperative paresis of the vocal folds. On the third postoperative day, the voice was impaired by all criteria of the GRBAS scale, but mainly due to roughness (85\%). Sixth month postoperatively, $62 \%$ of the subjects considered the voice to be altered. Asthenia was observed in $39 \%$. On the third postoperative day indirect laryngoscopy revealed the unchanged vocal folds, the edema and the shortening of one of the vocal folds in $56 \%, 42 \%$, and $1.9 \%$. Six months postoperatively, the vocal folds returned to their original form. Indirect laryngoscopy and ultrasonography had $100 \%$ concordance in assessing the mobility of the vocal folds. Patients with edema of the vocal folds had a significantly higher mean GRBAS grade than patients without edema. The mean GRBAS score decreased from 3.36 to 0.90, 3 days and 6 months, postoperatively. Voice alteration after thyroidectomy is always present. Postoperative edema represents a likely main cause of voice alteration and resolves within 6 months. Ultrasonography is recommended as alternative to indirect laryngoscopy in assessing of the vocal folds after thyroidectomy.
\end{abstract}

Keywords GRBAS score · Laryngoscopy · Postoperative voice alteration · Thyroidectomy · Ultrasonography

Airazat M. Kazaryan

kazaryan@gmail.com

1 Department of Faculty Surgery No 2, I. M. Sechenov First Moscow State Medical University, Moscow, Russia

2 Department of Plastic Surgery, Clinic Sirius, Moscow, Russia

3 Department of Gastrointestinal Surgery, Østfold Hospital Trust, Grålum, Norway

4 Intervention Centre, Oslo University Hospital Rikshospitalet, Oslo, Norway

5 Department of Surgery, Fonna Hospital Trust, Odda, Norway

6 Department of Surgery No 1, Yerevan State Medical University After M. Heratsi, Yerevan, Armenia

\section{Introduction}

If patients do not use their voice in a professional capacity, mild disturbances after thyroid surgery are often underestimated. Nevertheless, this does not eliminate the problem, especially after thyroidectomy.

Thyroidectomy is an operation of choice for most diseases of the thyroid gland with the laryngeal nerve injury rate within $0.5-1 \%$ in expert centers. However, obvious or subjective marked voice alteration is a frequent problem after surgery in patients with mobile vocal folds verified by laryngoscopy. Even mild voice alterations can cause significant discomfort for patients. This is particularly true for those with a profession associated with speech or singing, because these alterations can lead to temporary or permanent disability, which has both clinical and social significance. 
Previous studies have shown that up to $30 \%$ of patients after thyroidectomy complain of voice alterations even in the absence of paresis or palsy of the vocal folds, confirmed by laryngoscopy [1-3]. The exact cause for that remains unknown; it is possible that voice disorders are associated with traction and stretching of the nerve accompanied by subsequent swelling. However, it was noted that some of these patients have asymmetry of the vocal folds due to shortening of one of them $[4,5]$. Indirect or direct laryngoscopy represents traditional methods for assessing the state of the vocal folds. Ultrasonography has recently been applied as an alternative, non-invasive method for evaluation of the vocal folds, but only few reports were published in this issue [6-10].

The goal of the study was to determine the violation of the voice function after thyroidectomy, using GRBAS questionnaire, to uncover the causes of postoperative voice disorders and estimate the role of ultrasonography in assessing the condition of the vocal folds.

\section{Patients and Methods}

\section{Patients}

The present prospective cohort study included female patients who underwent total thyroidectomy in period between January 2017 and January 2018 at the University Clinical Hospital N 4 affiliated with the Chair of Faculty Surgery No 2, I. M. Sechenov First Moscow State Medical University (Moscow, Russia).

One year inclusion period for the study was defined as a compromise between expected number of observations to provide sufficient statistical patient pool and realistic time frames available for patient collection for the study. Men were excluded to provide homogenous patient cohort for statistical analysis especially when the number of men was quite small to meaningfully contribute to a total number of patients available for analysis.

\section{Inclusion Criteria and GRBAS Scale}

We determined three preoperative criteria for including patients into the study: first - no changes and irregularities in the mobility of the vocal folds, determined by indirect laryngoscopy; second - no changes and irregularities in the mobility of the vocal folds, determined by ultrasonography; and third - the absence of voice disorders assessed by the patient on the GRBAS scale. The GRBAS questionnaire has long been used to assess voice quality [11-14]. The abbreviation of the questionnaire corresponds well to the following criteria for assessing the voice (components of voice alterations): $\mathrm{G}$ (grade), alternative designation - dysphonia or quality; R (roughness) - hoarseness, rudeness or sharpness; B (breathiness) - breathing disorder or aspiration; A (asthenia) - fatigue or weakness; S (strain) - loudness, tension or tone. Patient gives an estimate for each criterion from 0 to 3 as follows: 0 - the norm, 1 - mild violation, 2 - moderate violation, and 3 - severe violation. The higher the score is, the more pronounced the voice violations are.

\section{Operative Techniques, Laryngoscopy and Ultrasonography}

Total thyroidectomies were performed extrafascially. The recurrent laryngeal nerves were visualized in all cases bilaterally. No anatomic variations were found. Ultrasonography and indirect laryngoscopy were applied as objective tools to assess mobility of the vocal folds. Indirect laryngoscopy was performed by the traditional method, using a standard mirror and reflector. Ultrasonography of the vocal folds was performed using B-scale ultrasound scanner (Esaote, Genova, Italy) using linear or sector probe with a frequency of 5.5 to $10 \mathrm{MHz}$. The technique of transcutaneous ultrasonography of the vocal folds consisted of their direct visualization in the area of their projection on the front surface of the neck with transverse scanning at the level of the middle of the thyroid cartilage, measurement of antero-posterior dimensions (length) of the vocal folds, evaluation of their mobility, symmetry and width of the glottis. The normal (non-affected) vocal folds are defined as two symmetrically arranged front-to-back hyperechoic strips of 2-4 mm wide, forming a triangle facing the back of the base (Fig. 1). When breathing or pronouncing the long sound " $i$ :" (corresponding to the long pronouncement of the English vowel "e") their movement is clearly visible. No more than a minute is required to visualize and assess the mobility of vocal folds.

Laryngoscopy, ultrasonographies and evaluation of the voice quality on the GRBAS scale were performed by different physicians in the blinded design, i.e. investigators were not knowledgeable about outcome of other assessment modality. Performers of laryngoscopy and ultrasonography did not know the patients' GRBAS score, i.e. assessors were blinded to the GRBAS score when assessing the vocal folds.

\section{Follow-up and Statistics}

We performed indirect laryngoscopy, ultrasonography of the vocal folds, as well as evaluation of the voice quality on the GRBAS scale before surgery, as well as 3 days, 2 months, and 6 months after surgery. Presence of voice alteration, grades of GRBAS scale as well as its particular components were compared 3 days, 2 months, and 6 months after surgery.

The data are presented as mean and standard deviation (SD), or number (percentage). The chi-squared test was used 
Fig. 1 Ultrasonography image of the normal (non-affected) vocal folds. 1 - the right vocal fold, 2 - the left vocal fold

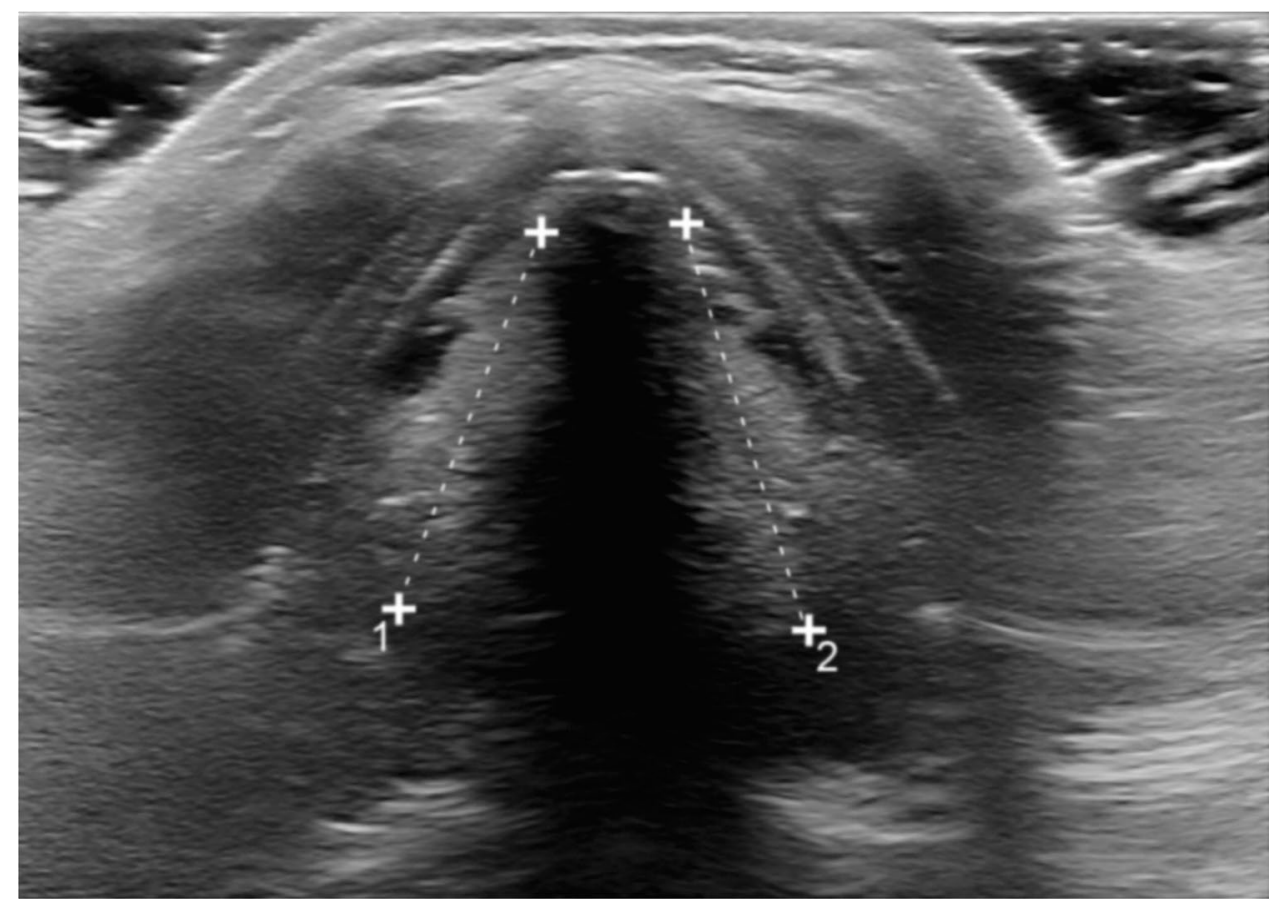

to compare frequencies. The analysis of variance test was used to compare used to compare continuous variables.

\section{Results}

There were 54 female patients aged from 24 to 77 years (mean age 53.4, SD 14.8 years) who satisfied the inclusion criteria. Ultrasonography has measured the length of the vocal folds to be from 14 to $24 \mathrm{~mm}$ (mean length 18.2 , SD26 mm), the width of the glottis was from 12 to $17 \mathrm{~mm}$ (mean width 14.1, SD $1.2 \mathrm{~mm}$ ). The vocal folds in all cases were symmetrical, and mobile when pronouncing the sound "I", which corresponds well to the ultrasound norm. When assessing the GRBAS scale, the score for all patients was 0 . The indications for surgery were diffuse toxic goiter in 7 (13\%) patients, proliferating colloid goiter in 17 (31.5\%) patients, follicular adenoma in $10(18.5 \%)$ cases, autoimmune thyroiditis in $2(3.7 \%)$ patients and thyroid cancer in $18(33.3 \%)$ patients. The latter group included 14 patients with papillary cancer, 3 patients with follicular cancer, and 1 patient with medullary cancer.

The patients were divided into 2 groups according to the results of the indirect laryngoscopy and ultrasonography on the 3rd day after surgery. In group I there were 52 patients with fully preserved vocal folds mobility after surgery. Group II consisted of 2 patients with obvious signs of paresis of one of the vocal folds, i.e. paramedian position and limited mobility. These two patients were operated for $\mathrm{T} 2$ and $\mathrm{T} 3$ thyroid cancer without any registered intraoperative events.

There were no missing data. None of the patients were missed from follow-up controls up to 6 months, postoperatively.

\section{Results of Examination of Group 1}

\section{Voice Function}

The results of our study demonstrated that the patients undergoing thyroidectomy have different degrees of severity of voice alteration even in the absence of postoperative paresis of the vocal folds, with their full mobility (Table 1). As it can be seen from Table 1, on the third day after surgery all the patients had a worse voice on all criteria of the GRBAS scale mainly due to roughness (84.6\%), grade $(46.2 \%)$, and strain $(42.3 \%)$. The asthenia and breathiness components were present in $34.6 \%$ and $15.4 \%$ observations.

The voice function improved in later terms after the surgery. Two and 6 months later, $76.9 \%$ and $61.5 \%$ of the examined subjects considered that their voice was altered. The component grade improved significantly shortly after operation, being present in only $7.7 \%$ and zero observations in two and 6 months, respectively; whereas the component of roughness improved less slowly, it was still present in $40.4 \%$ patients in 2 months; nevertheless, it remained only in $13.5 \%$ of observations in 6 months.

The strain and breathiness components have improved slowly and evenly over time, they were present in $19.2 \%$ 
Table 1 The frequency of voice impairment, assessed on the GRBAS scale, in 52 patients after thyroidectomy with mobile vocal folds (Group I)

\begin{tabular}{llll}
\hline Parameters & \multicolumn{3}{l}{$\begin{array}{l}\text { Number of the patients } \\
\text { at different time intervals after thyroidectomy }\end{array}$} \\
\cline { 2 - 4 } & 3 days & 2 months & 6 months \\
\hline Scale & 52 & 40 & 32 \\
GRBAS & $(100 \%)$ & $(76.9 \%)$ & $(61.5 \%)$ \\
G & 24 & 4 & 0 \\
grade & $(46.2 \%)$ & $(7.7 \%)$ & \\
R & 44 & 21 & 7 \\
roughness & $(84.6 \%)$ & $(40.4 \%)$ & $(13.5 \%)$ \\
A & 18 & 22 & 20 \\
asthenia & $(34.6 \%)$ & $(42.3 \%)$ & $(38.5 \%)$ \\
B & 8 & 7 & 4 \\
breathiness & $(15.4 \%)$ & $(13.5 \%)$ & $(7.7 \%)$ \\
S & 22 & 15 & 10 \\
strain & $(42.3 \%)$ & $(28.8 \%)$ & $(19.2 \%)$ \\
\hline
\end{tabular}

Data are presented in numbers (percentage)

and $7.7 \%$ in 6 months, respectively. The asthenia was a constant factor that did not significantly improved over time, it was present in $38.5 \%$ of observations in 6 months and was the main component responsible for still alternated voice in 6 months.

Figure 2 shows the average GRBAS score voice quality before and after thyroidectomy. The poorest GRBAS score is given on the third day after surgery (3.36 SD1.12), mainly due to roughness (1.46 SD0.68), as well as grade (0.66 SD0.52) and strain (0.66 SD0.57). Two and 6 months after the surgery, the average GRBAS score was reduced to
1.73 SD1.13 and 0.90 SD0.86, which corresponds well to an improvement in voice quality. The component of roughness leveled at 6 months (0.15 SD0.39), at the same time strain intensified (0.19 SD0.37) and asthenia became the leading cause of voice alteration (0.47 SD0.58).

\section{Indirect laryngoscopy and ultrasound}

According to the indirect laryngoscopy and ultrasonography on the third day after surgery, the vocal folds were completely unchanged and had the same appearance as before only in $29(55.8 \%)$ cases out of 52 patients in group 1. In $22(42.3 \%)$ cases, there was symmetrical edema of the vocal folds, and in $1(1.9 \%)$ patient, one of the vocal folds was shortened by $3 \mathrm{~mm}$ (approximate visual estimation). Two months after thyroidectomy, edema of the vocal folds resolved itself in most observations - 50 out of $52(96.2 \%)$ patients. Edema remained only in $2(3.8 \%)$ cases; one case was combined with shortening of one of the vocal folds. Six months after surgery, the vocal folds in all the patients returned to original form (Table 2). On the third day after thyroidectomy, indirect laryngoscopy and ultrasonography revealed no change (compared to preoperative evaluation) to the vocal folds in $29(55.8 \%)$ cases. In $22(42.3 \%)$ patients with edema revealed by laryngoscopy and ultrasonography on the third postoperative day, blurriness and vagueness of the vocal folds' contours were verified, which retained only in $1(1.9 \%)$ case 2 months later. In another $1(1.9 \%)$ case, indirect laryngoscopy and ultrasonography found a shortening of one of the vocal folds on the background of vagueness of vocal folds contours, which persisted even 2 months after

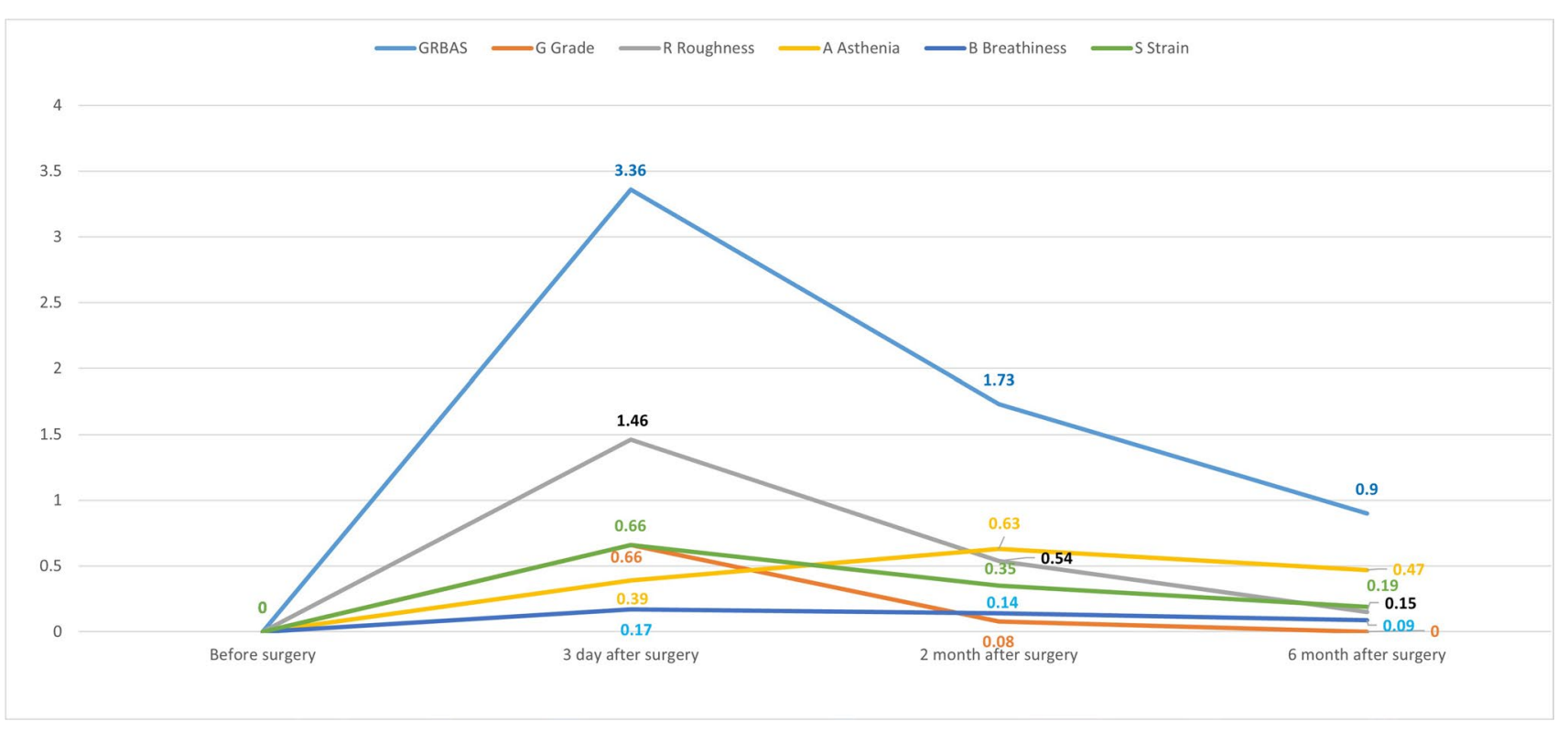

Fig. 2 Mean GRBAS voice quality before and after surgery 
Table 2 Condition of the mobile folds assessed by objective instrumental methods (ultrasonography and indirect laryngoscopy) and corresponding GRBAS scores in 52 patients at different time intervals after thyroidectomy

\begin{tabular}{|c|c|c|c|}
\hline \multirow[t]{2}{*}{$\begin{array}{l}\text { Condition of the vocal folds assessed by ultra- } \\
\text { sonography and indirect laryngoscopy }\end{array}$} & \multicolumn{3}{|c|}{$\begin{array}{l}\text { The sum of GRBAS scores at different time intervals } \\
\text { after thyroidectomy and patient number }\end{array}$} \\
\hline & 3 days & 2 months & 6 months \\
\hline Folds are not changed & $\begin{array}{c}3 \pm 1.16 \\
(n=29 / 55.8 \%)\end{array}$ & $\begin{array}{l}1,64 \pm 1.17 \\
(n=50)\end{array}$ & $\begin{array}{l}0.90 \pm 0.86 \\
(n=52)\end{array}$ \\
\hline Edema & $\begin{array}{l}3.77 \pm 1.11 \\
(n=22 / 42.3 \%)\end{array}$ & $\begin{array}{c}3 \\
(n=1 / 1.9 \%)\end{array}$ & $\begin{array}{l}- \\
(n=0)\end{array}$ \\
\hline Edema plus shortening of the vocal fold & $\begin{array}{c}5 \\
(n=1 / 1.9 \%)\end{array}$ & $\begin{array}{c}5 \\
(n=1 / 1.9 \%)\end{array}$ & $\begin{array}{l}- \\
(n=0)\end{array}$ \\
\hline Total & $(n=52)^{3.36 \pm 1.12}$ & $(n=52)^{1.73 \pm 1.13}$ & $\begin{array}{l}0.90 \pm 0.86 \\
(n=52)\end{array}$ \\
\hline
\end{tabular}

Data are presented in mean values ( \pm standard deviation) surgery. Both indirect laryngoscopy and ultrasonography recorded full mobility of the vocal folds. In 6 months after the operation, sonographic images of the vocal folds were the same as preoperatively.

Thus, the ultrasound image corresponded fully with the results of indirect laryngoscopy, which has been considered as a gold standard for evaluation of the mobility of the vocal folds (14). Hence, ultrasonography and indirect laryngoscopy had $100 \%$ concordance in assessing the mobility of the vocal folds.

The blurriness and vagueness of the vocal folds' contours are associated with their edema, which in most cases (96.2\%) resolved itself 2 months after thyroidectomy and the vocal folds returned to its original form.

\section{The Voice Function in Comparison with the Results of Indirect Laryngoscopy and Ultrasonography}

We compared the voice function in patients of group 1 with the results of indirect laryngoscopy and ultrasonography (Table 2). Patients who had edema of the vocal folds on the third postoperative day, graded their voice on the GRBAS scale much poorer compared with patients without edema (3.77 SD1.11 vs 3 SD1.16) $(p=0.021)$. The maximum score on the GRBAS scale 5 was in a patient with an identified shortening of one of the vocal folds in the presence of edema. Edema was resolved in most cases within 2 months and voice improves significantly $(P<0.001)$. The overall mean values of the GRBAS scale decreased from 3.36 SD1.12 to 1.73 SD1.13 than further to 0.90 SD0.86 from the 3 rd postoperative day to 2 and 6 months postoperatively, respectively $(P<0.001)$.

\section{Results of Examination of Patients of Group 2}

Indirect laryngoscopy performed on the third postoperative day revealed the limitation of mobility and the paramedian position of one of the vocal folds in 2 patients. Ultrasonography confirmed paresis, one of the vocal folds moved hardly with breathing and at pronouncement of the long sound "i:", it was positioned closer to the middle line. As a result, the glottis was narrowed up to 6 and $7 \mathrm{~mm}$. There were no respiratory disorders in these patients; however, there were pronounced voice alterations in all criteria of the GRBAS scale. On the third postoperative day, the scores were 8 and 9 in these two patients. The high score was persistent both in 2 ( 6 points) and in 6 months ( 5 points) after the surgery, this indicates a significant deterioration of the voice. The mobility of the vocal folds was restored in 2 and in 6 months after surgery verified by indirect laryngoscopy and ultrasonography, but not in full volume. This confirms the persistent paresis and causes voice dysfunction.

\section{Discussion}

Our series shows that all patients after thyroidectomy complain of temporary worsening of voice in the absence of paresis or palsy of the vocal folds. Moreover, in the early period after the surgery prevails roughness, grade and a decrease in strain, and in the late terms prevails increased voice asthenia. Other studies confirm the similar voice alterations after thyroidectomy with a rate of up to $30 \%$ [1-3]. Thus, even in the absence of paresis of the vocal folds, patients noted a deterioration of voice quality after thyroidectomy.

We showed that voice deterioration after total thyroidectomy in $42.3 \%$ of cases is likely due to edema of the vocal folds, which was likely due to intraoperative injury, i.e. manipulation of the trachea and larynx by the surgeon. In 2 months after surgery, $96.2 \%$ of patients had no edema and voice improved on all components of the GRBAS scale. The GRBAS score decreased from 3.77 SD1.11 on the third postoperative day to 1.64 SD1.17 in 2 months. Thus, persistent edema of the vocal folds represents a cause of postoperative voice alterations in cases with complete mobility of the vocal folds. 
The most pronounced voice alteration after thyroidectomy was on the third postoperative day in a patient with a shortened vocal fold in the background of edema. Previously, other authors also reported that voice disorders after thyroidectomy may be due to the shortening of the vocal folds $[4,5]$. The shortening is, probably, associated with pronounced edematous inflammatory changes, as a result of which the voice fold is tightened or contracted. In our opinion, the edema of the vocal folds, developed due to the injury during operation itself, i.e. manipulation of the surgeon on the trachea and larynx.

We found voice alterations in the absence of vocal fold edema after thyroidectomy in $55.8 \%$ of cases. Probably, voice disturbances can be due to the traction of the laryngeal nerve or to the intubation itself. According to other authors, post-intubational voice disorders are observed with a frequency of $6-11 \%$ [15], and traction and hyperextension of the laryngeal nerve during surgery are considered as the main reasons of its damage $[16,17]$. However, these questions require further study.

Thus, voice disturbances after thyroidectomy in the absence of paresis of the vocal folds are likely due to postoperative edema or, possibly, to the intubation itself or to the hyperextension of the laryngeal nerve. Whereas these series have shown clear association between edema of the vocal folds and the roughness component of the voice alteration, factors responsible for the persistent asthenia component are unknown [18]. The asthenia component of postoperative voice alteration was observed in one-fifth of the patients in our series without any improvement during the observation period of 6 months. Thus, further studies in this topic are urgent to be conducted in order to prevent or effectively treat this frequent side effect after total thyroidectomy.

We would like to emphasize he importance of preoperative evaluation of the vocal folds in patients referred to total thyroidectomy. Since voice alterations have been shown to be self-reported by all patients who undergo total thyroidectomy in our series, thus these groups of patients are disposed to bring informal or legal complains to hospital or surgeon. This represents an additional legal factor to justify the necessity of postoperative evaluation of the vocal folds to enable its comparison to their preoperative status. This will allow to define whether voice alterations are due to paresis or palsy of the vocal folds related to the recurrent laryngeal nerve injury or are due to other reasons not related to intraoperative nerve injury (presumably oedema of the vocal folds).

Indirect or direct laryngoscopies are the traditional methods of examining the vocal folds [14]. Ultrasonography is currently not widely used to assess the vocal folds' mobility and only a few authors use it for these purposes [6-9]. Our data demonstrate $100 \%$ concordance of ultrasonography and indirect laryngoscopy in assessing the mobility of the vocal folds. Other authors also testify high diagnostic efficiency of ultrasonography $(91.4 \%)$ in assessing the state of the vocal folds when compared with laryngoscopy in patients after thyroidectomy [9]. Considering the fact that the method is non-invasive and is quickly executed, ultrasonography, in our opinion, can be recommended as a routine method for assessing the vocal folds in patients both, before and after thyroidectomy.

This study has its limitations. It includes 54 cases which appeared to build up sufficient statistical pool to analyses voice alterations in cases of uncomplicated postoperative course. However, the cases with postoperative paresis of the vocal folds were present in two cases only, so the outcomes of this study cannot be generalized for patients with postoperative paresis of the vocal folds. Nevertheless, investigation of voice alteration after total thyroidectomy complicated with paresis of the vocal folds is an important topic of thyroid surgery [19]. Such study with application of ultrasonography of vocal folds, laryngoscopy and GRBAS scale will require multicenter design to provide sufficient statistical pool for research analysis. The study was performed in the academic hospital with middle volume of thyroid surgery; thus, outcomes cannot be directly generalized to low- and high-volume hospitals in regard to thyroid surgery.

\section{Conclusions}

All patients after thyroidectomy observed alterations in their voice in the absence of laryngeal nerve injury. Postoperative edema of the vocal folds is a likely reason for the voice worsening in the $42.3 \%$ of cases. Possibly, voice disturbances can also be due to the hyperextension of the laryngeal nerve a result of its traction or to the intubation itself. Ultrasonography is an adequate alternative to indirect laryngoscopy in the assessment of the vocal folds in patients with thyroid disease.

Author Contribution All authors have made substantial contributions to research design, as well as have read and approved the final manuscript. Besides that, all authors have participated in the following parts of the manuscript preparation:

Idea-AMS, VIS

Data acquisition-VIS, JAB, ARP

Data analysis and interpretation-AMS, VIS, JAB, ARP, AMK

Manuscript drafting-VIS, AMK

Critical revision-AMS, VIS, AYM, EGO, JAB, ARP, GTM, AMK

Funding Open access funding provided by University of Oslo (incl Oslo University Hospital). 


\section{Declarations}

Ethics Approval and Consent to Participate The authors are accountable for all aspects of the work. The protocol has been approved by the institutional review board (protocol reference number 7 , from 12.09.2016).

Conflict of Interest The authors declare no competing interests.

Open Access This article is licensed under a Creative Commons Attribution 4.0 International License, which permits use, sharing, adaptation, distribution and reproduction in any medium or format, as long as you give appropriate credit to the original author(s) and the source, provide a link to the Creative Commons licence, and indicate if changes were made. The images or other third party material in this article are included in the article's Creative Commons licence, unless indicated otherwise in a credit line to the material. If material is not included in the article's Creative Commons licence and your intended use is not permitted by statutory regulation or exceeds the permitted use, you will need to obtain permission directly from the copyright holder. To view a copy of this licence, visit http://creativecommons.org/licenses/by/4.0/.

\section{References}

1. De Pedro NI, Fae A, Vartanian JG, Barros AP, Correia LM, Toledo RN et al (2006) Voice and vocal self-assessment after thyroidectomy. Head Neck 28:1106-1114

2. Stojadinovic A, Shaha AR, Orlikoff RF, Nissan A, Kornak MF, Singh B et al (2002) Prospective functional voice assessment in patients undergoing thyroid surgery. Ann Surg 236:823-832

3. Lee JC, Breen D, Scott A, Grodski S, Yeung M, Johnson W, Serpell J (2016) Quantitative study of voice dysfunction after thyroidectomy. Surgery 160(6):1576-1581

4. Eckley CA, Sataloff RT, Hawkshaw M, Spiegel JR, Mandel S (1998) Voice range in superior laryngeal nerve paresis and paralysis. J Voice 12:340-348

5. Tsai V, Celmer A, Berke GS, Chhetri DK (2007) Videostroboscopic findings in unilateral superior laryngeal nerve paralysis and paresis. Otolaryngol Head Neck Surg 136:660-662

6. Wong KP, Lang BHH, Ng SH, Cheung CY, Chan CTY, Chan MY (2014) Is vocal cord asymmetry seen on transcutaneous laryngeal ultrasonography a significant predictor of voice quality changes after thyroidectomy? World J Surg 38:607-613

7. Kundra P, Kumar K, Allampalli V, Anathkrishnan R, Gopalakrishnan S, Elangovan S (2012) Use of ultrasound to assess superior and recurrent laryngeal nerve function immediately after thyroid surgery. Anaesthesia 67:301-302

8. Vetshev PS, Yankin PL, Zhivotov BA, Poddubny EI, Drozhzhin $\mathrm{AYu}$, Bondarev NS et al (2016) Ultrasound examination of the larynx in the diagnosis of mobility disorders of the vocal folds. Endokninnaia Kirurgiia 10(3):5-14 ([in Russian])

9. Makaryin VA, Uspenskaya AA, Timofeeva NI, Sleptsov IV, Semenov AA, Chernikov RA et al (2015) The use of percutaneous ultrasonography of the larynx to assess the mobility of vocal folds after performing surgical interventions on the thyroid and parathyroid glands. Klinicheskaia i Eksperimentalnaia Tiroidologiia 11(3):60-67 ([in Russian])

10. Shulutko AM, Semikov VI, Gorbacheva AV, Patalova AR, Boblak YA, Kulikov IO, Osmanov EG, Mansurova GT (2019) Ultrasound assessment of vocal cords function after thyroid surgery. Khirurgiia (Mosk) 7:24-28 ([in Russian])

11. Hirano M (1981) Clinical examination of voice. Springer, New York

12. Wuyts FL, De Bodt MS, Van de Heyning PH (1999) Is the reliability of a visual analogue scale higher than an ordinal scale? An experiment with the GRBAS scale for the perceptual evaluation of dysphonia. J Voice 13:508-517

13. Murri T, Medrado R, Hogikyan ND, Aviv JE (2004) The relationship between the quality of life. J Voice 18:183-192

14. Collins SR (2014) Direct and indirect laryngoscopy: equipment and techniques. Respir Care 59(6):850-862

15. Myssiorek D (2004) Recurrent laryngeal nerve paralysis: anatomy and etiology. Otolaryngol Clin North Am 37(1):25-44

16. Snyder SK, Lairmore TC, Hendricks JC, Roberts JW (2008) Elucidating mechanisms of recurrent laryngeal nerve injury during thyroidectomy and parathyroidectomy. J Am Coll Surg 206(1):123-130

17. Dionigi G, Wu CW, Kim HY, Rausei S, Boni L, Chiang FY (2016) Severity of recurrent laryngeal nerve injury in thyroid surgery. World J Surg 40(6): 1373-1381

18. Scerrino G, Melfa G, Raspanti C, Attard A, Mazzola S, Gullo R, Bonventre S, Attard M, Cocorullo G, Gulotta G (2017) The prevalence of post-thyroidectomy chronic asthenia: a prospective cohort study. Langenbecks Arch Surg 402(7):1095-1102

19. Lee DY, Lee KJ, Hwang SM, Oh KH, Cho JG, Baek SK, Kwon SY, Woo JS, Jung KY (2017) Analysis of temporal change in voice quality after thyroidectomy: single-institution prospective study. J Voice 31(2):195-201

Publisher's Note Springer Nature remains neutral with regard to jurisdictional claims in published maps and institutional affiliations. 